\title{
Outcome of ACL Reconstruction for Chronic ACL Injury in Knees without the Posterior Horn of the Medial Meniscus: Comparison with ACL Reconstructed Knees with An Intact Medial Meniscus
}

\author{
Kevin Syam, MS, Devendra K. Chouhan, MS, and Mandeep Singh Dhillon, MS, FRCS \\ Department of Orthopaedics, Post Graduate Institute of Medical Education and Research, Chandigarh, India
}

\begin{abstract}
Purpose: Cadaveric studies have shown that deficiency of the posterior horn of the medial meniscus (PHMM) increases strain on the anterior cruciate ligament (ACL) graft. However, its influence on the clinical and radiological outcome after ACL reconstruction is less discussed and hence evaluated in this study.

Materials and Methods: This study included 77 cases of ACL reconstruction with a minimum 18-month follow-up. Of the 77 cases, 41 patients with intact menisci were compared clinically and radiologically with 36 patients with an injury to the PHMM that required various grades of meniscectomy. The knees were evaluated using subjective International Knee Documentation Committee (IKDC) score and Orthopadische Arbeitsgruppe Knie (OAK) score.

Results: Cases with intact menisci showed better stability $(\mathrm{p}=0.004)$ at an average of 44.51 months after surgery. No significant differences were noted in the overall OAK score, subjective IKDC score, and functional OAK score $(\mathrm{p}=0.082, \mathrm{p}=0.526$, and $\mathrm{p}=0.363$, respectively). The incidence of radiological osteoarthrosis was significantly higher in the posterior horn deficient knees $(\mathrm{p}=0.022)$.

Conclusions: The tendency toward relatively higher objective instability and increased incidence of osteoarthrosis in the group with absent posterior horn reinforces its importance as a secondary stabiliser of the knee.
\end{abstract}

Keywords: Knee, Anterior cruciate ligament, Medial meniscus, Tear

\section{Introduction}

The anterior cruciate ligament (ACL) is the primary restraint to anterior translation of the tibia ${ }^{1)}$, augmented by the door stop phenomenon of the posterior horn of the medial meniscus $(\mathrm{PHMM})^{2-6)}$. Associated injuries to the medial meniscus in cases with chronic ACL injury are not infrequent in clinical practice.

Received December 8, 2016; Revised February 1, 2017

Accepted February 1, 2017

Correspondence to: Mandeep Singh Dhillon, MS, FRCS

Department of Orthopaedics, Post Graduate Institute of Medical

Education and Research, Chandigarh 160012, India

Tel: +91-172-269-4774, Fax: +91-172-275-6743

E-mail: drdhillon@gmail.com

This is an Open Access article distributed under the terms of the Creative Commons Attribution Non-Commercial License (http://creativecommons.org/licenses/by-nc/4.0/) which permits unrestricted non-commercial use, distribution, and reproduction in any medium, provided the original work is properly cited.
The reported incidence is about $61 \%$ and three quarters of these cases have the injury localised to the posterior horn, which necessitates removal of the torn posterior horn during ACL reconstruction in a good number of patients ${ }^{7-9)}$. The incidence of associated meniscal tears in chronic ACL injured knees is probably higher in Indian population where patients seek treatment very late.

Papageorgiou et al. ${ }^{3)}$ suggested that the interplay between the ACL graft and the medial meniscus is so substantial that the loss of medial meniscus leads to a $33 \%-50 \%$ increase in strain on the ACL graft. A routine literature review showed a number of experimental and clinical studies focussing on the implications of medial meniscectomy in ACL reconstructed knees ${ }^{3,4,10-15)}$. An absence of a normally functioning meniscus leads to higher instrumented laxity and stresses on the articular cartilage predisposing to early osteoarthritis $(\mathrm{OA})^{12,16,17)}$. However these studies did not specifically evaluate deficiency of any specific portion of the me- 
dial meniscus, particularly the PHMM. The importance of the PHMM is very well documented in experimental and cadaveric studies but evidence on its clinical relevance is lacking. The need for focussed studies to clarify the clinical implications of sacrificing PHMM during ACL reconstructions in chronic ACL injuries is obvious.

The aim of the present study was to find evidence in support of the clinical importance of preserving the PHMM to improve the outcome after ACL reconstruction for chronic ACL injuries. The knees that had ACL reconstruction for chronic ACL injury in the past were evaluated using International Knee Documentation Committee (IKDC) score, Orthopadische Arbeitsgruppe Knie (OAK) score, clinical joint laxity test and radiological evaluation of OA changes. These clinical and radiological outcome measures were compared between two groups of patients who underwent ACL reconstruction: one group with an intact medial meniscus and the other group with the PHMM sacrificed during ACL reconstruction.

\section{Materials and Methods}

\section{Study Material}

This was a retrospective study on 77 patients who had undergone ACL reconstruction during the period from January 2006 to 2012 for chronic ACL injury (with a minimum of 18 months of follow-up). Injuries older than a year at the time of ACL reconstruction were considered as chronic injuries. The present study analysed findings in these patients at the time when they were called for a follow-up evaluation as part of the study.

Ethical clearance was obtained prior to this study and selection of cases was done after reviewing the records of 457 cases who had undergone ACL reconstruction. Exclusion criteria were bilateral knee involvement, other ligament injuries, lateral meniscus injury, meniscal root injury, bucket handle tears, significant body tears of the medial meniscus, meniscal repair, inflammatory pathology, knees with more than physiological varus/valgus deformity, BMI $<19 \mathrm{~kg} / \mathrm{m}^{2}$ and $>35 \mathrm{~kg} / \mathrm{m}^{2}$, preoperative radiological OA changes and/or significant degenerative changes of the cartilage at the time of primary ACL reconstruction (greater than grade 2 Outerbridge scale ${ }^{18)}$ ).

Out of the 110 patients selected after exclusion, only 84 could be contacted either through a phone call, email or letter. Seven patients refused to come for review due to distant residence; ultimately, 77 patients gave informed consent to be included in the study. These 77 patients were divided into two groups: group 1 with 41 patients having an intact medial meniscus and group 2 with 36 patients having an isolated tear of the PHMM in whom the posterior horn was removed.

\section{Surgical Method and Follow-up}

All surgeries were performed by the same senior consultant (MSD), who had documented the intraoperative status of the menisci and cartilage. Diagnostic arthroscopy was performed first and those cases found to have tear in the posterior horn had partial or subtotal meniscectomy done, based on the type and extent of the tear. Complex chronic irreparable tears at the red-red or red-white zone underwent subtotal meniscectomy whereas tears in the white-white zone had partial meniscectomy. Of the 36 cases in group 2, partial meniscectomy was done in 14 and subtotal meniscectomy in 22. Bone-patellar tendon-bone graft was harvested and ACL reconstruction was done using the transtibial technique. Fixation of both ends of the graft was done using bioabsorbable interference screws after manual tensioning of the graft. An accelerated ACL rehabilitation protocol was followed in all cases and the follow-up protocol was implemented weekly in the 1st month, fortnightly in the 2nd month, monthly up to 6 months, 3 monthly up to 12 months and yearly thereafter.

\section{Outcome Analysis}

Patients included in the study had evaluation of knees done using the OAK score ${ }^{19)}$ and the 2000 IKDC subjective knee evaluation form ${ }^{20)}$. Clinical tests for residual laxity were conducted in the form of the anterior drawer test, Lachman test and pivot shift test by two physical therapy and rehabilitation physicians, who were blinded to the patient's preoperative and surgical data. Grading of these manual laxity tests were based on the article by Jensen $^{21)}$. The Lachman and anterior drawer tests were graded as grade 0 (no translation compared to the normal contralateral knee), grade 1 (translation greater than the contralateral knee but less than $5 \mathrm{~mm}$ ), grade 2 (6-10 $\mathrm{mm}$ translation) and grade 3 (greater than $10 \mathrm{~mm}$ translation). The pivot shift test was graded as grade 0 (absent), grade 1 (glide), grade 2 (abrupt reduction) and grade 3 (momentary locking). For radiological assessment, the standard weight bearing anteroposterior view and the lateral view in $60^{\circ}$ of knee flexion were obtained. Radiological grading of OA changes was done using Kellgren and Lawrence (K-L) grading system for OA knees ${ }^{22)}$.

\section{Statistical Methods}

Analysis of the data was done using SPSS ver. 17.0 (SPSS Inc., Chicago, IL, USA). Normal-Quantile plots were constructed in order to examine whether the data was normally distributed or 
not. After the normality was established, the functional OAK score, the K-L radiological OA grading outcomes and the clinical instability test results of both groups were compared using chisquare test. The IKDC subjective scores between the two groups were compared using $t$-test. The other non-parametric variables were compared using Mann-Whitney $U$ test. Statistical significance was accepted when the $p$-value was $<0.05$.

\section{Results}

The age of the patients, delay in treatment after ACL injury, follow-up period, preoperative IKDC and OAK scores were comparable between the groups (Table 1).

\section{OAK and Stability}

The outcome evaluated using the OAK score was excellent in 27 out of 41 cases in group 1 and 16 out of 36 in group 2. The difference was not found to be statistically significant $(\mathrm{p}=0.082)$ (Table 2 ).

The manual Lachman test for objective stability assessment

Table 1. Comparison of Age, Delay in Treatment, Follow-up Duration and Preoperative Scores between Groups

\begin{tabular}{lccc}
\hline \multicolumn{1}{c}{ Parameter } & $\begin{array}{c}\text { PHMM } \\
\text { intact (n=41) }\end{array}$ & $\begin{array}{c}\text { PHMM } \\
\text { absent (n=36) }\end{array}$ & p-value \\
\hline $\begin{array}{l}\text { Average age (yr) } \\
\text { Delay in treatment after } \\
\text { ACL injury (mo) }\end{array}$ & 30.15 & 30.44 & 0.871 \\
$\quad \begin{array}{l}\text { Follow-up duration after } \\
\text { surgery (mo) }\end{array}$ & 44.51 & 41.33 & 0.544 \\
$\begin{array}{c}\text { Average preoperative } \\
\text { subjective IKDC score }\end{array}$ & 74.71 & 70.97 & 0.322 \\
$\begin{array}{c}\text { Preoperative overall OAK } \\
\text { score (moderate/bad) }\end{array}$ & $36 / 5$ & $28 / 8$ & 0.876 \\
\hline
\end{tabular}

PHMM: posterior horn of the medial meniscus, ACL: anterior cruciate ligament, IKDC: International Knee Documentation Committee, OAK: Orthopadische Arbeitsgruppe Knie.

Table 2. Excellent Outcomes according to the OAK Category in Both Groups

\begin{tabular}{cccc}
\hline $\begin{array}{c}\text { OAK } \\
\text { category }\end{array}$ & $\begin{array}{c}\text { Group 1 with } \\
\text { excellent score }\end{array}$ & $\begin{array}{c}\text { Group 2 with } \\
\text { excellent score }\end{array}$ & p-value \\
\hline Overall OAK & 27 & 16 & 0.082 \\
Category A & 39 & 35 & 1.000 \\
Category B & 38 & 36 & 0.243 \\
Category C & 38 & 22 & 0.004 \\
Category D & 35 & 29 & 0.363 \\
\hline
\end{tabular}

OAK: Orthopadische Arbeitsgruppe Knie. revealed that 25 out of 36 in group 2 had grade 1 laxity whereas only 13 out of 41 had grade 1 laxity in group 1, showing a statistically significant difference between the groups $(\mathrm{p}=0.001)$ (Table 3). It is pertinent to note, however, that none of the patients had more than grade 1 laxity in the Lachman test and a positive Lachman test was not associated with the overall OAK score and the category D (functional) outcomes.

Group 2 had 5 cases with grade 2 and more Anterior drawer positivity while group 1 had none ( $\mathrm{p}=0.019$ ). On the pivot shift test, 3 cases had a grade 1 positive pivot shift test in group 1 and 12 cases had in group $2(\mathrm{p}=0.041)$. None of the patients in either group had a grade 2 or 3 pivot shift test or an episode of give way after ACL reconstruction (Table 3). But among those with a positive pivot shift, no statistically significant difference was seen in the overall OAK score and category D outcome.

\section{Subjective IKDC Score}

The mean subjective IKDC score was 89.51 in group 1 and 87.80 in group $2(\mathrm{p}=0.526)$ (Table 4$)$. Cases with positive joint laxity in both groups did not show statistically significant difference in the subjective IKDC score.

Table 3. Restuls of Clinical Instability Tests in Both Groups

\begin{tabular}{lcccc}
\hline \multicolumn{1}{c}{ Clinical instability test } & $\begin{array}{c}\text { Group 1 } \\
\text { with laxity }\end{array}$ & $\begin{array}{c}\text { Group 2 } \\
\text { with laxity }\end{array}$ & p-value \\
\hline Lachman test & 13 & 25 & 0.001 \\
Anterior Drawer test grade 2 and more & 0 & 5 & 0.019 \\
Pivot shift test grade 1 & 3 & 12 & 0.041 \\
\hline
\end{tabular}

Table 4. Average Subjective IKDC Scores of Both Groups

\begin{tabular}{lcccc}
\hline \multicolumn{1}{c}{ Group } & No. & $\begin{array}{c}\text { IKDC score } \\
\text { (mean) }\end{array}$ & $\begin{array}{c}\text { Standard } \\
\text { deviation }\end{array}$ & $\begin{array}{c}\text { Standard } \\
\text { error (mean) }\end{array}$ \\
\hline Group 1 & 41 & 89.51 & 12.65 & 1.98 \\
Group 2 & 36 & 87.80 & 10.63 & 1.77 \\
Positive Lachman test & & & & \\
$\quad$ Group 1 & 13 & 91.69 & 8.40 & 2.33 \\
Group 2 & 25 & 85.33 & 11.55 & 2.31 \\
Positive anterior drawer test & & & & \\
Group 1 & 0 & 0 & 0 & 0 \\
$\quad$ Group 2 & 5 & 84.74 & 12.58 & 2.96 \\
Positive pivot shift test & & & & \\
Group 1 & 3 & 89.32 & 13.50 & 3.61 \\
Group 2 & 12 & 87.08 & 11.23 & 2.45 \\
\hline
\end{tabular}

IKDC: International Knee Documentation Committee. 


\section{Radiographic Osteoarthritis}

Group 1 had only 4 cases of radiological OA (K-L grade 2 and more) at an average follow-up of 44.51 months whereas group 2 had 12 cases at an average follow-up of 41.33 months $(\mathrm{p}=0.022)$. On the comparison of chronicity of the cases with OA, the delay from injury to treatment was an average of 7.5 years in group 1 and 4.5 years in group 2 , showing a statistically significant difference $(p=0.003)$. The follow-up period for cases with OA was comparable between group 1 and group 2 (43.19 months vs. 41.04 months; $\mathrm{p}=0.068$ ).

\section{Discussion}

The purpose of this study was to investigate whether preservation of the PHMM helps to improve the outcome of ACL reconstruction. ACL reconstructed knees where the PHMM had to be sacrificed were compared with ACL reconstructed knees having intact medial menisci. The present study revealed statistically significant differences between the groups in terms of laxity and the incidence of radiological OA, both being high in the group where the PHMM was sacrificed. The overall OAK score difference between two groups showed only a trend towards significance.

It has been observed that ACL reconstruction does not always achieve an ideal outcome, and this has been attributed to the various associated risk factors such as meniscal injury, chondral lesions, other ligamentous injuries, severity of initial trauma, chronicity of injury and age ${ }^{15,16,23)}$. Existing studies have variable reports regarding the prevalence of knee OA (range, 10\% to $90 \%)^{12,13,17,24,25)}$. Studies have tried to establish the complex relation between the medial meniscus and the ACL graft and effects of medial meniscectomy on ACL reconstructed knees ${ }^{3,410-15)}$. However, these studies are not comparable to our study with regard to the selection of cases. The present study used strict selection criteria and specifically focused on the functional importance of the PHMM in ACL reconstructed knees.

The outcome parameters including pain and swelling (category A in OAK scoring), objective range of motion and strength (category B outcome), subjective IKDC score and overall OAK score did not show a statistically significant difference between the two groups in the present study. This is in contrast to the findings in the study by Shelbourne and Gray ${ }^{10)}$ where 928 patients were followed for 8.6 years after ACL reconstruction. They found a significant difference in the mean subjective IKDC score between the medial meniscectomy group and without meniscectomy group. This difference could be attributed to the longer followup in the study by Shelbourne and Gray ${ }^{10)}$. Moreover, their study included the meniscus removal group for comparison as opposed to the present study involving the group where meniscectomy was localized to the posterior horn. Some other studies have also found significant differences in scores with regard to pain, effusion and functional outcome, in contrast to the findings of the present study ${ }^{11,15)}$. Heterogeneity of the cases in those studies could be the reason for this contradiction. The overall OAK scores showed only a trend towards significance in the present study, with the $\mathrm{p}$-value nearing the significance level $(\mathrm{p}=0.082)$, suggesting the possibility of having poorer outcome in the group with absent PHMM. The significantly different outcome score found by Shelbourne and Gray ${ }^{10)}$ could be due to the different outcome measure in a broad heterogeneous meniscus removal group, the larger sample size and/or the longer follow-up in their study. Their study group included cases of either or both meniscus injuries and patients who had revision surgery for meniscus injury after ACL reconstruction. There were a significantly large number of cases with lateral meniscus injury and both meniscus injury in their study.

With regard to stability, the results of present study corroborate with the findings in the study by Shelbourne and Gray ${ }^{10)}$, who found a significant difference in instrumented laxity. Manual laxity tests were utilised in the present study and category $\mathrm{C}$ outcome in OAK score (objective stability) showed a significant difference, supporting the concept that there is a significant stabilising role for the PHMM in ACL reconstructed knees. On the other hand, some authors have failed to find a significant difference in stability between the meniscectomy group and the meniscus intact group, which is probably due to the fact that cases selected were very heterogeneous ${ }^{11,13,14)}$.

As with previous published studies, the present study also showed a significantly increased prevalence of radiological OA in the PHMM deficient group ${ }^{10-12,17,25)}$. Moreover, the incidence of OA in group 2 was higher, even though the average injuryto-surgery duration was significantly shorter in this group in comparison to group 1. The higher incidence of laxity and OA in group 2 at average follow-up of 41.33 months could be the result of complex interplay of multiple factors. Firstly, the sacrifice of the posterior horn would have resulted in the loss of the secondary stabilising effect, which hypothetically led to the cascade of adverse mechanical milieu for the articular cartilage and the healing ACL graft ${ }^{3}$. Secondly, increased contact stress on the articular cartilage resulting from the loss of meniscal tissue could have also contributed to the higher rate of $\mathrm{OA}^{26,27)}$.

The absence of statistically significant difference in subjective IKDC scores between the two groups raises doubt regarding 
the actual relevance of demonstrable objective laxity when the patients have no subjective instability or episode of give way. Double bundle or extra-articular reconstruction aimed at reducing postoperative laxity have also failed to produce clinically significant benefits ${ }^{28-30}$. This raises questions regarding the clinical relevance of preserving the PHMM for achieving objective stability. It is possible that the increased contact stress after meniscectomy, rather than the stabilizing effect of the PHMM, has a more important role to play in causing early OA in ACL reconstructed knees without PHMM.

The present study has limitations: the retrospective study design, the relatively small sample size and the absence of instrumented test for measuring laxity. However, the subjective IKDC score, which does not have any objective bias, failed to reveal any significant difference in our study. Moreover, instrumented laxity is also found to have flaws giving inconclusive results when used as an objective measure of laxity ${ }^{10,11,13,14)}$.

\section{Conclusions}

Even though it is difficult to draw definite conclusions from this study, we have found evidence supporting the secondary stabilizing role of the PHMM in the knee for improving the outcome of ACL reconstruction: the trend towards worsening clinical results and the significantly higher risk of instability and early radiological OA in the absence of PHMM at a relatively short follow-up after ACL reconstruction. Prospective randomised comparative studies involving a larger sample with a longer follow-up on the outcome after meniscal repair and meniscal replacement will shed more light on the importance of preserving the PHMM during ACL reconstruction in knees with chronic ACL injuries and will be of help in drafting guidelines for management of these complex injuries.

\section{Conflict of Interest}

No potential conflict of interest relevant to this article was reported.

\section{References}

1. Butler DL, Noyes FR, Grood ES. Ligamentous restraints to anterior-posterior drawer in the human knee: a biomechanical study. J Bone Joint Surg Am. 1980;62:259-70.

2. Levy IM, Torzilli PA, Warren RF. The effect of medial meniscectomy on anterior-posterior motion of the knee. J Bone
Joint Surg Am. 1982;64:883-8.

3. Papageorgiou CD, Gil JE, Kanamori A, Fenwick JA, Woo SL, Fu FH. The biomechanical interdependence between the anterior cruciate ligament replacement graft and the medial meniscus. Am J Sports Med. 2001;29:226-31.

4. Seon JK, Gadikota HR, Kozanek M, Oh LS, Gill TJ, Li G. The effect of anterior cruciate ligament reconstruction on kinematics of the knee with combined anterior cruciate ligament injury and subtotal medial meniscectomy: an in vitro robotic investigation. Arthroscopy. 2009;25:123-30.

5. Shoemaker SC, Markolf KL. The role of the meniscus in the anterior-posterior stability of the loaded anterior cruciatedeficient knee: effects of partial versus total excision. J Bone Joint Surg Am. 1986;68:71-9.

6. Spang JT, Dang AB, Mazzocca A, Rincon L, Obopilwe E, Beynnon B, Arciero RA. The effect of medial meniscectomy and meniscal allograft transplantation on knee and anterior cruciate ligament biomechanics. Arthroscopy. 2010;26:192201.

7. Cipolla M, Scala A, Gianni E, Puddu G. Different patterns of meniscal tears in acute anterior cruciate ligament (ACL) ruptures and in chronic ACL-deficient knees: classification, staging and timing of treatment. Knee Surg Sports Traumatol Arthrosc. 1995;3:130-4.

8. Henning CE. Current status of meniscus salvage. Clin Sports Med. 1990;9:567-76.

9. Smith JP 3rd, Barrett GR. Medial and lateral meniscal tear patterns in anterior cruciate ligament-deficient knees: a prospective analysis of 575 tears. Am J Sports Med. 2001;29:415-9.

10. Shelbourne KD, Gray T. Results of anterior cruciate ligament reconstruction based on meniscus and articular cartilage status at the time of surgery: five- to fifteen-year evaluations. Am J Sports Med. 2000;28:446-52.

11. Wu WH, Hackett T, Richmond JC. Effects of meniscal and articular surface status on knee stability, function, and symptoms after anterior cruciate ligament reconstruction: a longterm prospective study. Am J Sports Med. 2002;30:845-50.

12. Lohmander LS, Englund PM, Dahl LL, Roos EM. The longterm consequence of anterior cruciate ligament and meniscus injuries: osteoarthritis. Am J Sports Med. 2007;35:175669.

13. Cohen M, Amaro JT, Ejnisman B, Carvalho RT, Nakano KK, Peccin MS, Teixeira R, Laurino CF, Abdalla RJ. Anterior cruciate ligament reconstruction after 10 to 15 years: association between meniscectomy and osteoarthrosis. Arthroscopy. 2007;23:629-34. 
14. Cox CL, Huston LJ, Dunn WR, Reinke EK, Nwosu SK, Parker RD, Wright RW, Kaeding CC, Marx RG, Amendola A, McCarty EC, Spindler KP. Are articular cartilage lesions and meniscus tears predictive of IKDC, KOOS, and Marx activity level outcomes after anterior cruciate ligament reconstruction? A 6-year multicenter cohort study. Am J Sports Med. 2014;42:1058-67.

15. Kartus JT, Russell VJ, Salmon LJ, Magnusson LC, Brandsson S, Pehrsson NG, Pinczewski LA. Concomitant partial meniscectomy worsens outcome after arthroscopic anterior cruciate ligament reconstruction. Acta Orthop Scand. 2002; 73:179-85.

16. Louboutin H, Debarge R, Richou J, Selmi TA, Donell ST, Neyret P, Dubrana F. Osteoarthritis in patients with anterior cruciate ligament rupture: a review of risk factors. Knee. 2009;16:239-44.

17. Oiestad BE, Engebretsen L, Storheim K, Risberg MA. Knee osteoarthritis after anterior cruciate ligament injury: a systematic review. Am J Sports Med. 2009;37:1434-43.

18. Outerbridge RE. The etiology of chondromalacia patellae. J Bone Joint Surg Br. 1961;43:752-7.

19. Muller W, Biedert R, Hefti F, Jakob RP, Munzinger U, Stäubli HU. OAK knee evaluation: a new way to assess knee ligament injuries. Clin Orthop Relat Res. 1988;(232):37-50.

20. Hefti F, Muller W. Current state of evaluation of knee ligament lesions. The new IKDC knee evaluation form. Orthopade. 1993;22:351-62.

21. Jensen K. Manual laxity tests for anterior cruciate ligament injuries. J Orthop Sports Phys Ther. 1990;11:474-81.

22. Kellgren JH, Lawrence JS. Radiological assessment of osteoarthrosis. Ann Rheum Dis. 1957;16:494-502.

23. Barenius B, Ponzer S, Shalabi A, Bujak R, Norlen L, Eriksson $\mathrm{K}$. Increased risk of osteoarthritis after anterior cruciate ligament reconstruction: a 14-year follow-up study of a randomized controlled trial. Am J Sports Med. 2014;42:104957.

24. Oiestad BE, Holm I, Aune AK, Gunderson R, Myklebust G, Engebretsen L, Fosdahl MA, Risberg MA. Knee function and prevalence of knee osteoarthritis after anterior cruciate ligament reconstruction: a prospective study with 10 to 15 years of follow-up. Am J Sports Med. 2010;38:2201-10.

25. Gillquist J, Messner K. Anterior cruciate ligament reconstruction and the long-term incidence of gonarthrosis. Sports Med. 1999;27:143-56.

26. Walker PS, Erkman MJ. The role of the menisci in force transmission across the knee. Clin Orthop Relat Res. 1975; (109):184-92.

27. Fukubayashi T, Kurosawa $H$. The contact area and pressure distribution pattern of the knee: a study of normal and osteoarthrotic knee joints. Acta Orthop Scand. 1980;51:871-9.

28. Hussein M, van Eck CF, Cretnik A, Dinevski D, Fu FH. Prospective randomized clinical evaluation of conventional single-bundle, anatomic single-bundle, and anatomic doublebundle anterior cruciate ligament reconstruction: 281 cases with 3- to 5-year follow-up. Am J Sports Med. 2012;40:51220 .

29. Lee S, Kim H, Jang J, Seong SC, Lee MC. Comparison of anterior and rotatory laxity using navigation between singleand double-bundle ACL reconstruction: prospective randomized trial. Knee Surg Sports Traumatol Arthrosc. 2012; 20:752-61.

30. Branch T, Lavoie F, Guier C, Branch E, Lording T, Stinton S, Neyret P. Single-bundle ACL reconstruction with and without extra-articular reconstruction: evaluation with robotic lower leg rotation testing and patient satisfaction scores. Knee Surg Sports Traumatol Arthrosc. 2015;23:2882-91. 